\title{
A Wireless Location System in LTE Networks
}

\author{
Qi Liu, Rongyi Hu, and Shan Liu \\ Network Technology Research Institute, China Unicom, Beijing 100084, China \\ Correspondence should be addressed to Shan Liu; liushancandy@163.com
}

Received 29 September 2016; Revised 23 December 2016; Accepted 15 January 2017; Published 16 February 2017

Academic Editor: Xiaohong Jiang

Copyright (C) 2017 Qi Liu et al. This is an open access article distributed under the Creative Commons Attribution License, which permits unrestricted use, distribution, and reproduction in any medium, provided the original work is properly cited.

\begin{abstract}
Personal location technologies are becoming important with the rapid development of Mobile Internet services. In traditional cellular networks, the key problems of user location technologies are high-precision synchronization among different base stations, inflexible processing resources, and low accuracy positioning, especially for indoor environment. In this paper, a new LTE location system in Centralized Radio Access Network (C-RAN) is proposed, which makes channel and location measurement more available, allocation of baseband processing resources more flexible, and location service capability opening. The location system contains more than two antenna clusters, and each of them gets time-difference-of-arrival (TDOA) of sounding reference signals (SRSs) from different antennas. Then, based on data provided by location measurement units (LMUs), the location information server calculates TDOAs and derives the users' position. Furthermore, a new location algorithm is raised which can achieve distributed antennas collaboration and centralized location computing. And an improved optimized algorithm with the best TDOA selection is proposed. Finally, simulations are given out to verify the efficiency of the proposed algorithm in this LTE location system.
\end{abstract}

\section{Introduction}

In recent years, wireless location technologies, especially technologies for indoor environment, have attracted attention of many powerful companies including network operators. Although the Global Positioning System (GPS) could provide personal location services, the 3rd Generation Partner Project (3GPP) has also proposed user location projects in LTE networks. Particularly, terminals maybe cannot receive GPS signals in indoor environment due to the weak received signal and multipath reflection. As LTE networks are being deployed around the world, location technologies with LTE networks need to be studied further, which could enhance user location services in all different environments [1-3].

There are mainly two challenges in traditional LTE location system [4, 5]. One is the limitation of indoor networks architecture, causing the difficulties of distinguishing positioning signals from DAS (distributed antenna system), and time synchronization among different base stations. And the other is the low positioning accuracy due to the location algorithm and the complex LOS/NLOS communication environment $[6,7]$.
According to the study of previous work [8-11], timeof-arrival (TOA), time-difference-of-arrival (TDOA), and hybrid location algorithms based on time-related measurements are the research hotspot at present. Most location systems used to take TOA/TDOA techniques. However, few location algorithms or schemes are proposed for LTE systems with $20 \mathrm{MHz}$ bandwidth, especially for complex scenarios such as indoor positioning. So new studies or algorithms related to LTE systems are needed in order to improve positioning performances of cellular networks, especially location accuracy and implementation complexity $[12,13]$.

In this paper, a wireless location system in LTE networks based on C-RAN (Cloud Radio Access Networks) architecture is introduced [14-16]. To be specific, traditional cellular Radio Access Networks (RAN) usually consist of many standalone base stations. It is a proposed architecture for future cellular networks called C-RAN or Cloud-RAN, which allow distributed transmission of RF signals from remote radio heads (RRH), centralized processing of baseband signals in baseband units (BBU) pool, and real-time virtualization and cloud computing. For the cellular location services, these functions can better support dynamic computing resource 
allocation and flexible selection of RF antennas, which would guarantee both communication quality and positioning accuracy. Meanwhile, high-precision synchronization among different base stations for TDOA measurement is easily solved by involving the LMU (location measurement unit), which can independently measure and calculate the TDOAs of different antennas. By the way, both stand-alone LMU and base station with LMU function are supported in our system.

In this paper, we also propose a new method that calculates and analyzes the TDOAs of sounding reference signals (SRSs) in uplink channel of C-RAN. According to the TDOAs of SRS and classical nonlinear least squares (NLLS) algorithm, a new location algorithm based on the LTE location system is proposed. It combines information from different antenna clusters provided by LMUs, which does not require synchronization among BSs. In addition, we propose an improved algorithm by optimizing the selection of TDOAs in order to enhance the accuracy of the users' location. Both the experiment test and simulation results show the performance gain of the proposed algorithms and verify the efficiency.

The remainder of the article is organized as follows. A wireless location system in LTE networks is given in Section 2, including the system model, the performance of SRS correlation for TDOA estimation, and the proposed location algorithms. In Section 3, the simulation and test results are described. Finally, Section 4 concludes the paper.

\section{A Wireless Location System in LTE Networks}

2.1. System Model. Generally, in order to ensure the location performance, location systems need to be low-latency and high-precision, especially for multiuser systems. So the location procedure shall be completed in a short time (e.g., $10 \mathrm{~ms}$ ) in LTE networks, including channel measurement, largescale computing, and information feedback. Meanwhile, with the development of positioning technology, a software update of the related equipment comes naturally. Traditional cellular network architecture obviously cannot support these features. However, in C-RAN architecture, operators can rapidly deploy or upgrade their networks and make full use of base station and antennas resources.

In this section, we introduce the user location mechanism in LTE system with the C-RAN architecture, where Uplink-Time Difference of Arrival (U-TDOA) technology is applicative. Here, location measurement of SRSs and channel measurement functions are achieved in LMUs, RF antennas or RRHs are flexibly selected and managed, and location algorithms and computing are centrally realized in BBU pool. Figure 1 gives the LTE location system model in C-RAN architecture.

Firstly, $M(M \in \mathbb{N})$ receiver units are used for collecting the LTE signals from the target User Equipment (UE) or Mobile Terminals (MT). In other words, there are $M$ antenna clusters deployed and $N_{m}\left(N_{m} \in \mathbb{N}, N_{m} \geq 2\right)$ antennas in cluster- $m(m=1,2, \ldots, M)$, and the antenna gap is usually set as 1 to 10 meters for more accurate location measurement.
By the way, each location antenna cluster should contain at least two antennas to insure the available measurement of TOA/TDOA.

Secondly, there are $M$ wireless location measurement units (LMUs) which connect the location servers in this system. In cluster- $m$, a LMU receives SRSs from each antenna in this cluster, extracts the time differences of SRSs from different antennas, and calculates TDOAs. It is worth mentioning that there is no need for synchronization between different LMUs or base stations.

Thirdly, LMUs report location measurement data to the location information server, including TDOAs and related antenna clusters' information. The server collects time differences from $M$ clusters and gets user location information by the positioning algorithm. In this architecture, the time delay of location information transmitting will be less than traditional cellular architecture.

Figure 2 describes functions and signal processing in this location system. The RF front ends receive the LTE signals and transmit the signals to LMUs. LMUs process the baseband analog signals through Analog to Digital signals conversion (A/D) at first, then exact single user's SRS, and calculate TDOAs. Finally, location information server estimates the user's position through the location algorithm and stores the information in databases of BBU pool.

2.2. SRS Correlation Performances for TDOA Estimation. Two methods for TDOA estimation are widely used. One is calculating the cross-correlation between two received signals and getting TDOA [17]. But it does not work well in multipath environments especially for the indoor localization [18]. Another is estimating TOA at first by cross-correlation between the received signal and the transmitted pseudorandom sequence. And then the difference between the two TOA estimations is calculated, assuming that all receivers are synchronized. In this paper, TDOA is detected and estimated based on the second method, upon which the TDOA error model is built.

SRS is a reference signal in LTE networks. It is usually used to figure out the channel quality of uplink path. Mobile Terminals (MT) send SRS at the last symbol of a slot. A MT can transmit SRS signal every 2 subframes at the most and every 32 frames (320 subframes) at the least. A signaling parameter transmitted by eNodeB, named as SRS-ConfigIndex, tells UEs the periodicity of SRS transmission, and the period can be $2,5,10,20,40,80,160$, and 320 milliseconds (ms) [5]. SRS is generated by Zadoff-Chu sequences, which are good candidates as their ideal correlation properties.

In this section, we evaluate SRS correlation performance in LTE networks by simulations. In the simulations, SRS is generated by a MT. Then SRS and useful information data are combined in a frame according to 3GPP LTE standards. The eNodeB receives the uplink signals and maps frequency signals to time domain, obtaining the Single-Carrier Frequency Division Multiple Access (SC-FDMA) signals. Then the received signals are correlated with the local SRS, which is the same with SRS generated by the MT. So the correlation peak is found. The peak time is what we want to get for our location system, which is helpful for obtaining the accurate 


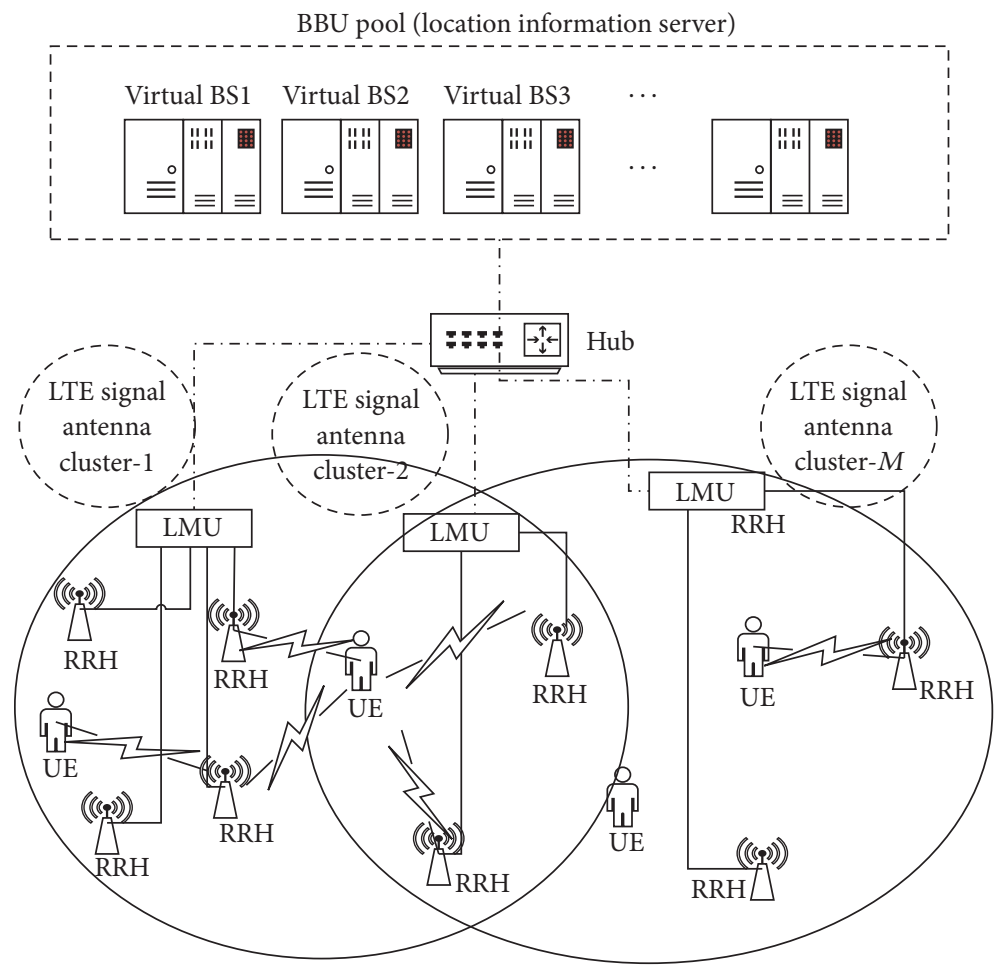

FIgURE 1: LTE location system in C-RAN architecture.

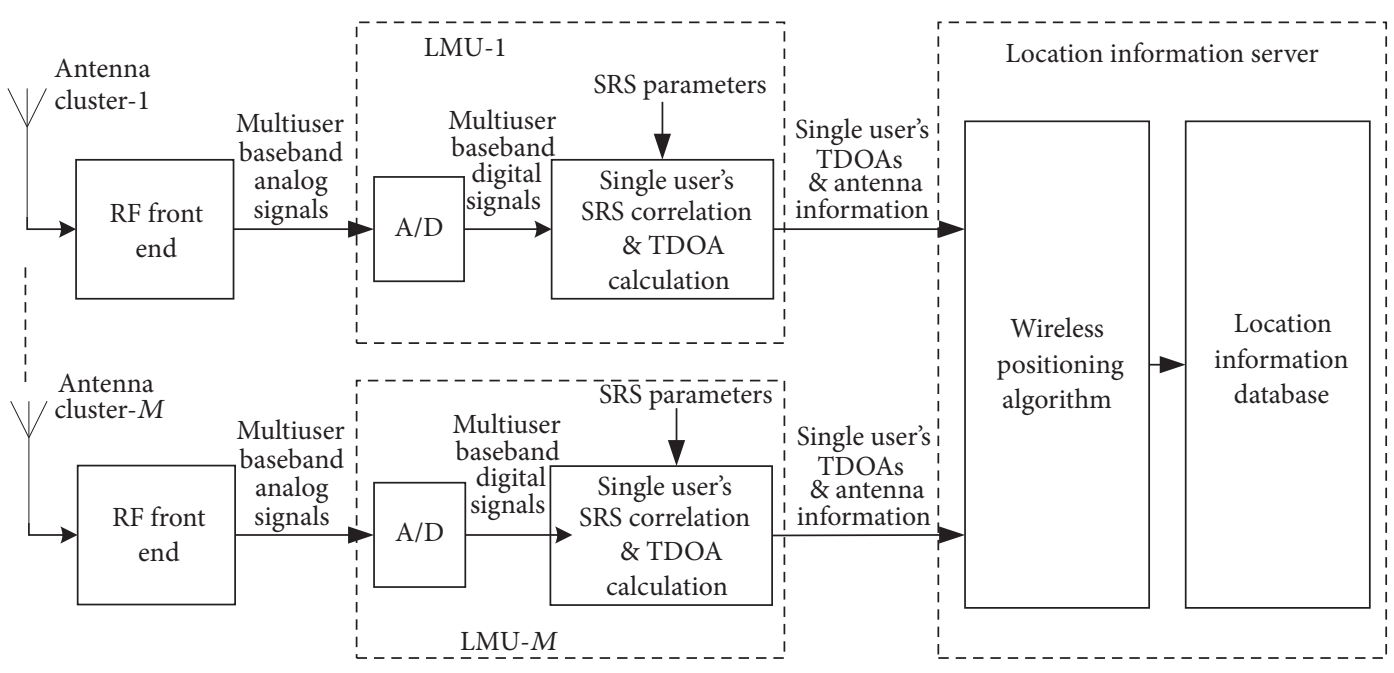

FIgURE 2: LTE location system function diagram.

TDOA. To be simplified, the Additive White Gaussian Noise (AWGN) channel model is applied in the simulations. And the default sampling rate is $30.72 \mathrm{MHz}$.

In LTE networks, SRSs from different users are transmitted by time-division mode or code-division mode. Their correlation results are shown in Figure 3. In time-division mode, as Figure 3(a) shows, SRS from a MT is easy to distinguish from others', which has a perfect performance of correlation peak. Then, we can easily get the TOAs and TDOAs of the different signals by detecting and analyzing their SRSs.
However, as Figure 3(b) shows, in the case of codedivision mode, there are more side peaks in correlation results. The time interval between each side peak is approximately $410 \mathrm{~ns}$. It is greater than the accuracy of time advance (TA), which is a parameter in LTE networks. So we can use TA to assist in finding the main peak time.

In addition, we can take advantage of oversampling before the SRS correlation, upon which the errors of time differences can be reduced. Figure 4 shows SRS correlation performance comparison between nonoversampling and oversampling. It is obvious that the correlation curve of oversampling is more 


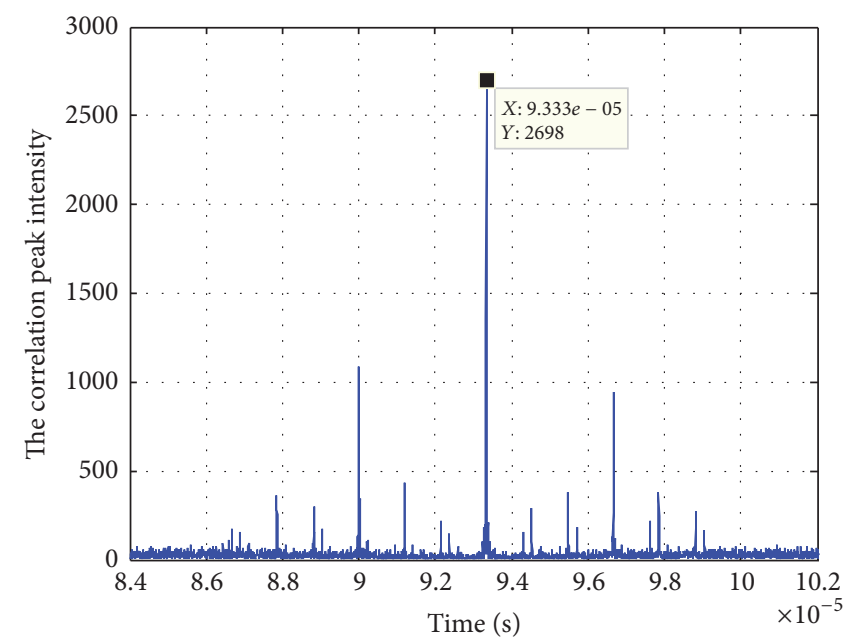

(a) Time-division mode

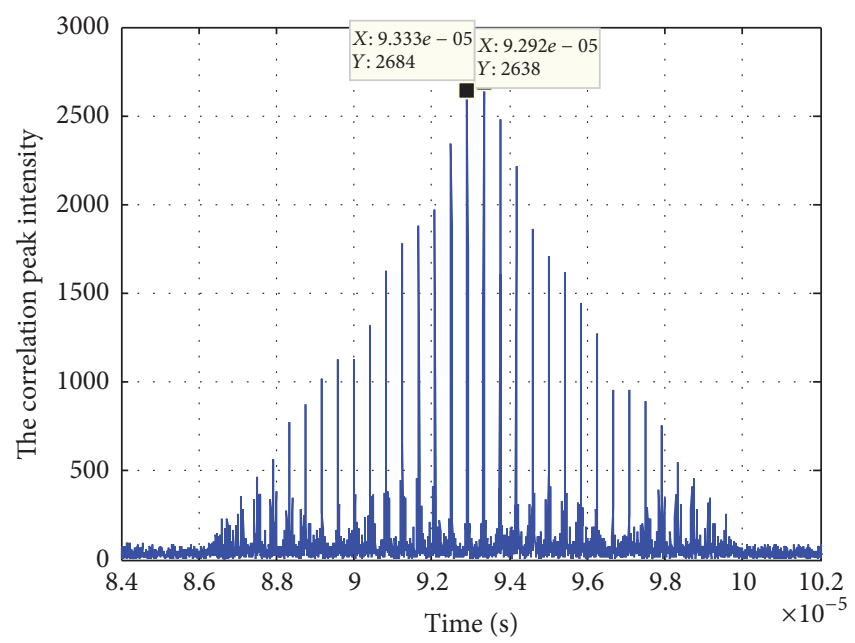

(b) Code-division mode

FIGURE 3: SRS correlation performance in time-division and code-division mode.

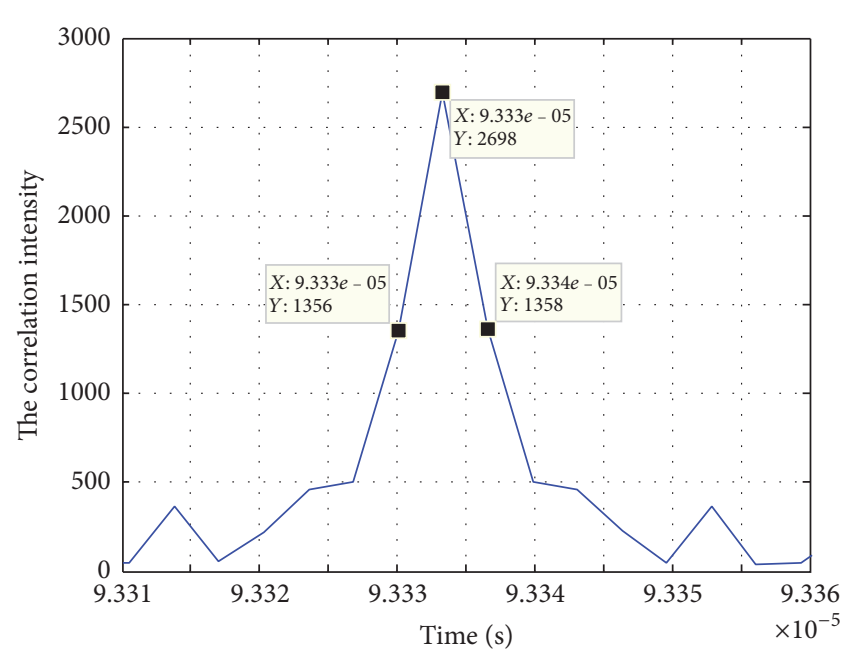

(a) Default sampling rate

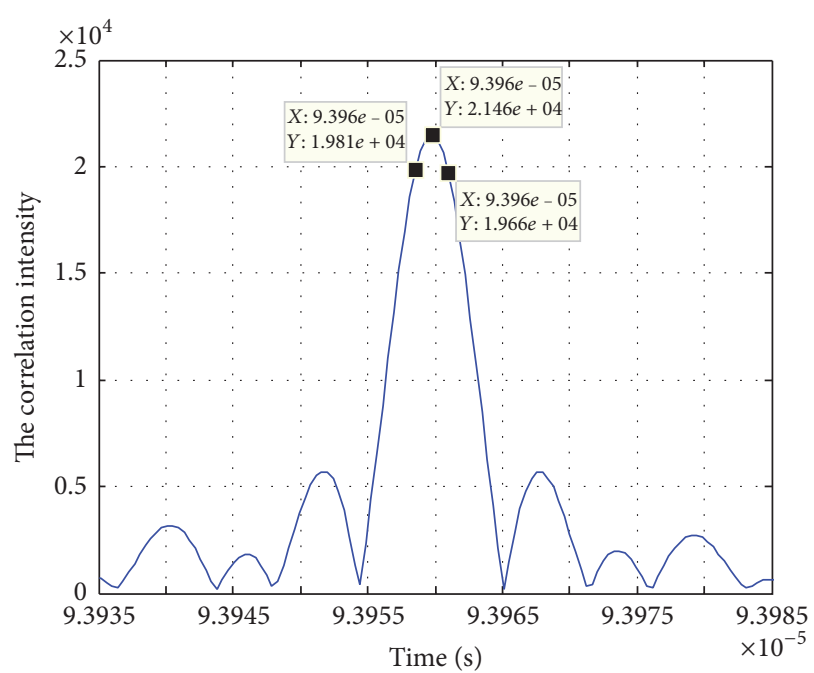

(b) Oversampling

FIGURE 4: SRS correlation performance comparison between nonoversampling and oversampling.

smooth, and this could increase the accuracy of peak time with increased intensity of sample points. So oversampling can be used for TDOA detection.

2.3. Location Algorithm. First, we assume one cluster with $N$ antennas in this location system, and $\mathbf{x}$ represents a MT's position to be estimated. $\left\|\mathbf{x}-\mathbf{x}_{n}\right\|$ is the true distance between antenna- $n$ and the MT, where $\mathbf{x}_{n}$ represents antenna$n$ 's position $n=1,2, \ldots, N$. Set antenna-1 as an anchor; then $h_{n}(\mathbf{x})=\left\|\mathbf{x}-\mathbf{x}_{n}\right\|-\left\|\mathbf{x}-\mathbf{x}_{1}\right\|$ represents the difference of distance. It is defined that $\mathbf{h}(\mathbf{x})=\left[h_{2}(\mathbf{x}), \ldots, h_{n}(\mathbf{x})\right]^{\mathrm{T}}$, where $[\cdot]^{\mathrm{T}}$ is the transpose operation. $\mathbf{r}$ represents measured difference of distance, shown as (1). Here, $\boldsymbol{\varepsilon}$ is measurement error and obeying normal distribution $\left(0, \sigma_{n}{ }^{2}\right)$.

$$
\mathbf{r}=\mathbf{h}(\mathbf{x})+\boldsymbol{\varepsilon} .
$$

$\mathbf{h}(\mathbf{x})$ is linearized about an initial reference position $\mathbf{x}_{0}$ ignoring high-order terms by Taylor series expansion, shown in (2). Here $\mathbf{H}_{0}$ is the Jacobian matrix of $\mathbf{h}(\mathbf{x})$ at $\mathbf{x}_{0}$.

$$
\mathbf{h}(\mathbf{x}) \approx \mathbf{h}\left(\mathbf{x}_{0}\right)+\mathbf{H}_{0} *\left(\mathbf{x}-\mathbf{x}_{0}\right) \text {. }
$$

So (1) can be expressed as follows:

$$
\begin{aligned}
& \mathbf{r}=\mathbf{y}+\mathbf{h}\left(\mathbf{x}_{0}\right)-\mathbf{H}_{0} \mathbf{x}_{0}, \\
& \mathbf{y}=\mathbf{H}_{0} \mathbf{x}+\boldsymbol{\varepsilon} .
\end{aligned}
$$

Here, $\mathbf{y}$ is a linearized approximation of $\mathbf{r}$. Then $\mathbf{x}$ can be calculated by least squares (LS) estimation method, shown in (4). Here, $\mathbf{R}$ is the covariance matrix of $\boldsymbol{\varepsilon}$, and $(\cdot)^{-1}$ is the inverse matrix operation.

$$
\widehat{\mathbf{x}}=\left(\mathbf{H}_{0}^{\mathrm{T}} \mathbf{R}^{-1} \mathbf{H}_{0}\right)^{-1} \mathbf{H}_{0}^{\mathrm{T}} \mathbf{R}^{-1} \mathbf{y} .
$$


In addition, the algorithm also extends to the scenario of $M$ clusters in the location system. Assuming that each cluster can have different number of antennas (at least 2), we can combine information getting from $M$ clusters to calculate the MT's position by (4), where $\mathbf{R}^{\prime}$ is the covariance matrix of $\boldsymbol{\varepsilon}^{\prime}$ :

$$
\begin{gathered}
\mathbf{y}^{\prime}=\left[\begin{array}{c}
\mathbf{y}_{1} \\
\mathbf{y}_{2} \\
\vdots \\
\mathbf{y}_{M}
\end{array}\right], \\
\mathbf{H}_{0}^{\prime}=\left[\begin{array}{c}
\mathbf{H}_{01} \\
\mathbf{H}_{02} \\
\vdots \\
\mathbf{H}_{0 M}
\end{array}\right], \\
\boldsymbol{\varepsilon}^{\prime}=\left[\begin{array}{c}
\boldsymbol{\varepsilon}_{1} \\
\boldsymbol{\varepsilon}_{2} \\
\vdots \\
\boldsymbol{\varepsilon}_{M}
\end{array}\right] .
\end{gathered}
$$

As the closed form of the estimation position cannot be derived, we apply an iterative method to minimize the error according to Taylor series expansion as follows. To be specific, the estimated position for the $k$ th iteration should be derived as

$$
\widehat{\mathbf{x}}(k)=\left(\mathbf{H}_{0}^{\prime \mathrm{T}} \mathbf{R}^{\prime-1} \mathbf{H}_{0}^{\prime}\right)^{-1} \mathbf{H}_{0}^{\prime \mathrm{T}} \mathbf{R}^{\prime-1} \mathbf{y}^{\prime} .
$$

Algorithm 1 (the proposed algorithm).

Step 1. Initialize the position $\mathbf{x}_{0}$, and calculate $\mathbf{y}^{\prime}, \mathbf{H}_{0}^{\prime}, \mathbf{R}^{\prime}$ with (3) and (5).

Step 2 (iteration). (1) Estimate $\widehat{\mathbf{x}}(k)$ with (5) and (6), where $k \in \mathbb{N}_{+}$and $\widehat{\mathbf{x}}(0)=\mathbf{x}_{0}$.

(2) If $\|\widehat{\mathbf{x}}(k)-\widehat{\mathbf{x}}(k-1)\| \leq \sigma$, stop; else, continue;

(3) Set $\mathbf{x}_{0}=\widehat{\mathbf{x}}(k)$; go to Step 1 .

Step 3. Return the final estimated position, until a preset number of iterations are reached or until convergence.

2.4. Improved Location Algorithm. In LTE systems, we cannot ensure the links between signal sources and receivers (or the receiving antenna clusters) are always in line of sight (LOS) due to the multipath. Though all TDOAs getting from $M$ clusters can be gathered together for calculation, the bad estimated TDOAs caused by NLOS or penetration may make the performance of the proposed Algorithm 1 worse.

To deal with the bias caused by bad estimation, we proposed an improved algorithm of TDOA selection, which is mainly based on $\mathrm{kNN}$ ( $k$-NearestNeighbor) algorithm, a nonparametric method used for classification and regression.
First, by kNN classification, each estimated position calculated by randomly divided TDOAs is classified by a majority vote of its neighbors, with the estimation being assigned to the class most common among its $k$ nearest neighbors ( $k$ is a positive integer, typically small). Particularly if $k=1$, it is simply assigned to the class of that single nearest neighbor. Then, by kNN regression (which is simplified as calculating the average of the values of $k$ nearest neighbors), the best estimated positions and their reliable TDOAs with property value are selected, and the worst ones are got rid of.

The detailed procedure of the improved algorithm of TDOA selection is as follows. Firstly, a coarse selection is used. Set an upper limit of TDOA ( $\left.T_{\text {upper }}\right)$ according to the maximum distance between receiving antennas in one cluster. Then, for all $N_{0}$ TDOAs, get rid of the obviously bad ones if

$$
\operatorname{TDOA}(i)>T_{\text {upper }}, \quad i=1,2, \ldots, N_{0} .
$$

Secondly, the remaining TDOAs, whose number is assumed as $N_{1}$, will be selected in an accurate way. Here to simplify, we denote TDOAs as $T(i), i=1,2, \ldots, N_{1}$. Choose $M\left(3 \leq M \leq N_{1}\right)$ of the TDOAs randomly,

$$
A_{1}, A_{2}, \ldots, A_{C_{N_{1}}^{M}} \subset\left\{T(1), T(2), \ldots, T\left(N_{1}\right)\right\},
$$

where $A_{i} \triangleq\left\{T\left(x_{i 1}\right), T\left(x_{i 2}\right), \ldots, T\left(x_{i M}\right)\right\}$ and $\left\{x_{i 1}, x_{i 2}, \ldots\right.$, $\left.x_{i M}\right\} \subset\left\{1,2, \ldots, N_{1}\right\}, i=1,2, \ldots, C_{N_{1}}^{M}$.

And calculate the predicted positions with (5) and (6) in Algorithm 1 for all $C_{N_{1}}^{M}$ cases, expressed as $\operatorname{pos}(i)(i=$ $\left.1,2, \ldots, C_{N_{1}}^{M}\right)$.

Then according to the distribution of positions, the range of all positions is divided into continuous $J(J \geq 4)$ equivalent segmented blocks. The cumulative probability of each segment $j(j=1,2, \ldots, J)$ is expressed as

$$
P(j)=\frac{\sum_{i=1}^{C_{N_{1}}^{M}} p_{j}(i)}{C_{N_{1}}^{M}}, \quad j=1,2, \ldots, J,
$$

where

$$
\begin{aligned}
p_{j}(i)=\left\{\begin{aligned}
1, & \operatorname{dis}(i) \\
0, & \operatorname{dis}(i) \notin \operatorname{segment}(j)
\end{aligned}\right. \\
\quad j=1,2, \ldots, J, \quad i=1,2, \ldots, C_{N_{1}}^{M} .
\end{aligned}
$$

Therefore, according to the cumulative probability distribution, we can get the densest segment, expressed as segment $\left(j_{\max }\right)$. Furthermore, the related positions in $\operatorname{segment}\left(j_{\max }\right)$ are obtained, whose number is added up as $L$. Here, $C_{N_{1}}^{M} / J<L \leq C_{N_{1}}^{M}$. Then the median position of $L$ points can be easily obtained, called point $A\left(\operatorname{pos}_{A}\right)$. The distance between the positions and point $A$ can be expressed as

$$
\operatorname{dis}(i)=\left\|\operatorname{pos}(i)-\operatorname{pos}_{A}\right\|, \quad i=1, \ldots, L .
$$

By sorting all these distances, the former $K(K \leq L)$ values can be achieved, which are considered as the nearest 
neighbor points and most likely estimated positions of target location. Here, $K$ depends on the size of sample data.

Finally, according to the $K$ nearest neighbor points, the related TDOAs for $\operatorname{pos}^{\prime}(k)(k=1,2, \ldots, K)$ are easy to achieve which are marked as $A_{j k}=\left\{T\left(x_{j 1}\right), T\left(x_{j 2}\right), \ldots\right.$, $\left.T\left(x_{j M}\right)\right\}$, where $A_{j k} \in\left\{A_{1}, A_{2}, \ldots, A_{C_{N_{1}}^{M}}\right\}, j \in\{1,2, \ldots$, $\left.C_{N_{1}}^{M}\right\}$. Then we can calculate the times of selected TDOAs as the weight of $\operatorname{TDOA}(i)$ :

$$
W(i)=\sum_{k=1}^{K} \sum_{m=1}^{M} w_{k, m}(i),
$$

where

$$
\begin{aligned}
& w_{k, m}(i)= \begin{cases}1, & \text { if } i=x_{m}(j), T(i) \in A(k) \\
0, & \text { if } i \neq x_{m}(j) \text { or } T(i) \notin A(k),\end{cases} \\
& m=1 \cdots M, k=1 \cdots K, \quad j=1,2, \ldots, C_{N_{1}}^{M}, i=1 \cdots N_{1} .
\end{aligned}
$$

By sorting the weights of these TDOAs, we can select the top $N_{2}\left(M \leq N_{2} \leq N_{1}\right)$ TDOAs, which are the optimized selection of TDOAs. Based on these TDOAs and Algorithm 1 above, we can recalculate the optimized estimated position.

The steps of the improved Algorithm 2 are as follows.

Algorithm 2 (the improved algorithm).

Step 1 (coarse selection). Set an upper limit of TDOA, and get rid of the bad TDOAs with (7).

Step 2 (accurate selection). (1) Calculate the positions with (5), (6), and (8) by random $M$ TDOAs for all $C_{N}^{M}$ cases.

(2) Calculate the cumulative probability of continuous $J$ segmented blocks with (9), and get the densest segment $j_{\max }$ and the related $L$ positions.

(3) Select the $K$ nearest neighbor points with (11) after resorting the distances.

(4) Weight the related TDOAs with (12), and select top $\mathrm{N}_{2}$ TDOAs.

Step 3. Calculate the final optimized position with the selected TDOAs using Algorithm 1.

\section{Simulation and Test Results}

3.1. Simulation Results. In this section, we mainly evaluate the performance of proposed location algorithms in the LTE location system through several simulations.

Some common simulation settings are as follows: SRS is configured in time-division mode among different users. To be simplified, the Additive White Gaussian Noise (AWGN) channel model is applied in the simulations. The default sampling rate is $30.72 \mathrm{MHz}$, and 10 times oversampling is used in this location system. We assume that the simulation scenario is a room whose size is $20 \mathrm{~m} \times 20 \mathrm{~m} \times 3 \mathrm{~m}$ and $c(c=1,2, \ldots, 5)$ antenna clusters are deployed in it. Each cluster contains $m(m=2,3,4)$ antennas.

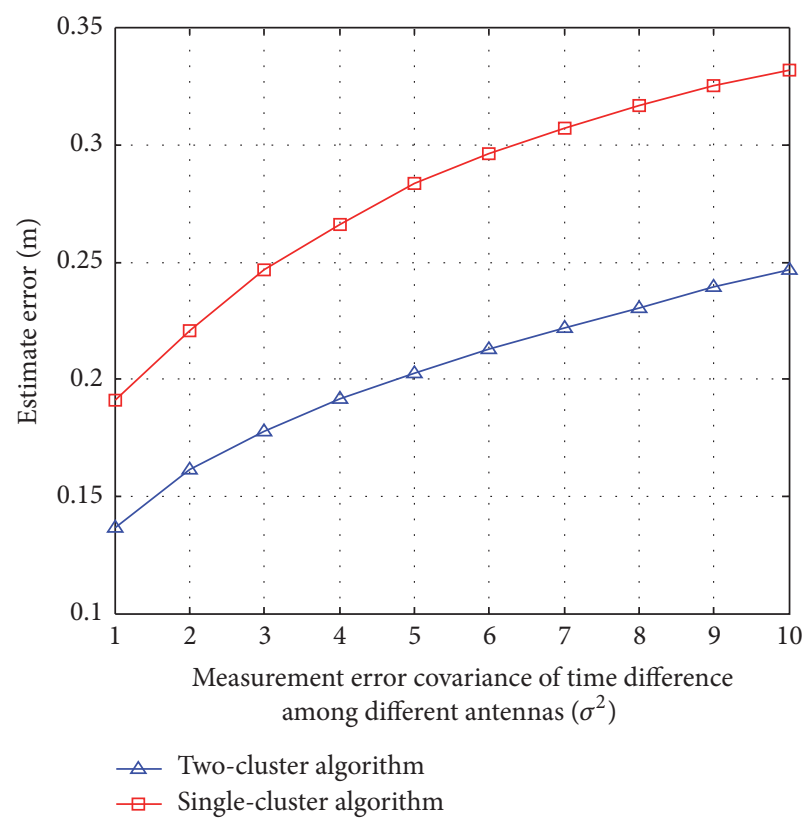

FIGURE 5: The performance of LTE location system between single cluster and 2 clusters.

In the first simulation group, users are randomly distributed. Firstly, performance comparisons between single cluster (with 4 antennas) and 2 clusters (one with 4 antennas and the other with 2 antennas) are given in Figure 5. The location accuracy of 2 clusters is better than that of single cluster. Its estimating error is improved about $25 \%$.

Furthermore, Figure 6 shows the cumulative distribution function (CDF) of estimation error with different antenna clusters $\left(\right.$ clust $\left._{N}\right)$, each of which has 4 antennas. Owing to the fact that simulation scenario (a room $20 \mathrm{~m} \times 20 \mathrm{~m} \times 3 \mathrm{~m}$ ) is limited, the performance of 1 cluster is not quite good but seems to be nearly perfect when there are above 2 antenna clusters deployed. It is easy to find that the estimation error goes smaller with antenna clusters number increasing, and the estimation error is controlled within 1.1 meters when there are more than 2 clusters. Particularly, for 5 clusters, the estimation error can be even all controlled below 0.5 meters. In some degree, the results show that the more antenna clusters can bring better performance.

In the second simulation group, we assumed that 2 clusters are used and the user/MT moves in a certain area among the clusters. The location performances are compared when the user is in different positions. As shown in Figure 7, as the user moves from the edge of the area to the center in a line, the location accuracy is obviously improved. For example, when the user moves at the center areas among the antenna clusters like $(3,1)$ or $(3.5,1)$, the estimating error is lower than 0.1 meters, rather better than that of the edge areas especially at $(0,1)$ and $(4.5,1)$.

In the last simulation group, assuming the MT randomly distributed, we give out the performance by the cumulative distribution function (CDF) of estimation error for different clusters (clust $\left._{N}=3,4,5\right)$. And the comparison between the proposed algorithms, that is, Algorithm 1 based on the 


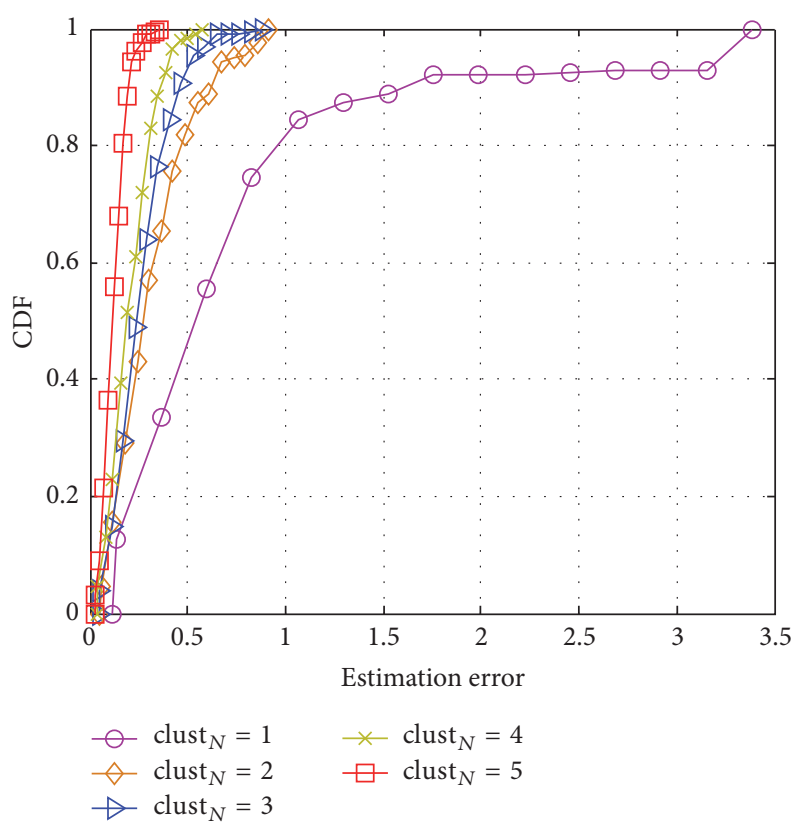

FIGURE 6: The performance of LTE location system with different antenna clusters, where clust $_{N}=1,2, \ldots, 5$.

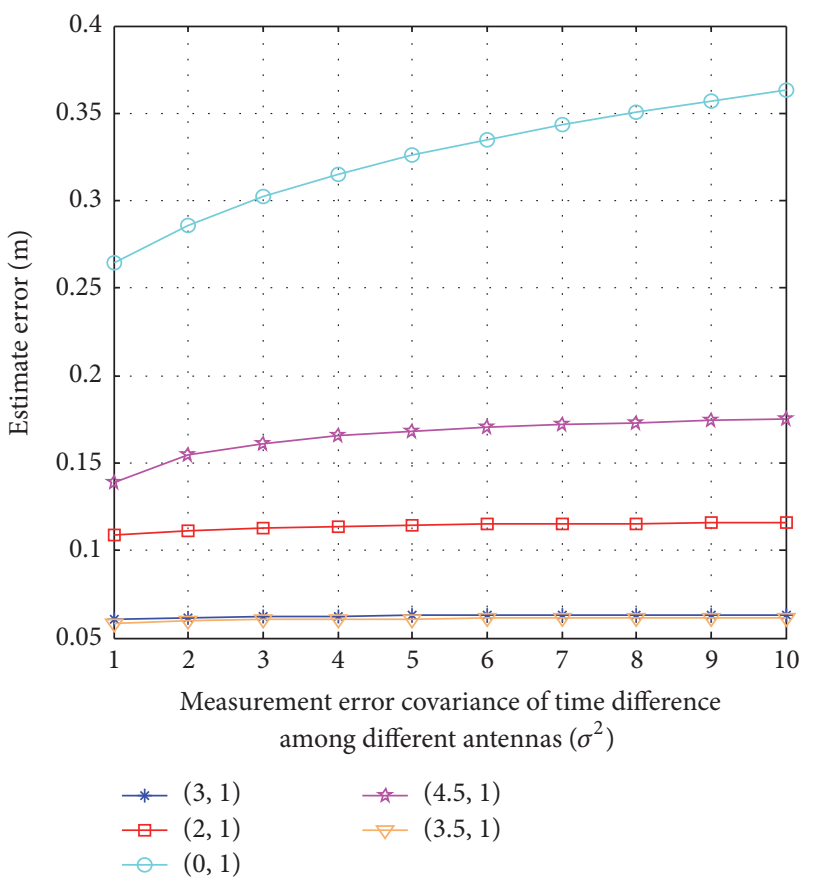

FIGURE 7: The performance for user in different positions in LTE location system.

weighted least square method and the improved Algorithm 2 based on TDOA selection, is also analyzed. Here, we assumed the good TDOAs $\left(\mathrm{N}_{2}\right)$ are mostly accounted for $75 \%$ of all $\left(N_{0}\right)$.

As shown in Figure 8, it is easy to find that the estimation error goes smaller with clusters number increasing for both location algorithms. Meanwhile, for all three cases with

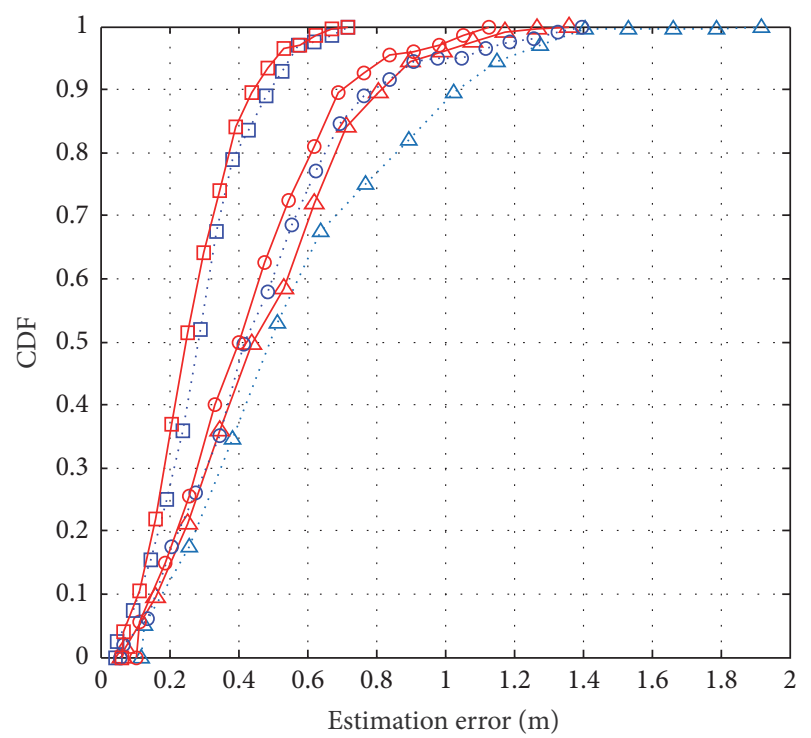

$$
\begin{aligned}
& \triangle \text { Algorithm 1, clust }{ }_{N}=3 \quad \odot \text { Algorithm 2, } \text { clust }_{N}=4 \\
& \triangle \text { Algorithm 2, clust }_{N}=3 \quad \cdots \boxminus \text { Algorithm 1, clust }{ }_{N}=5 \\
& \text { ○. Algorithm 1, clust } \text { Al }_{N}=4 \square \text { Algorithm 2, clust }_{N}=5
\end{aligned}
$$

FIGURE 8: The performance comparison of the proposed two algorithms, where clust ${ }_{N}=3,4,5$, and each has 4 antennas.

different clusters, the cumulative probability of estimation error with Algorithm 2 has a better performance, especially when there are 3 antenna clusters. At the expense of increased time complexity, Algorithm 2 can obviously improve the accuracy of location, which proves that the optimization of TDOA selection is effective and feasible.

3.2. Test Results. In order to testify the performance of the proposed system and algorithms, we have done some experiment in a conference room. The size of the room is $12.2 \mathrm{~m} *$ $17 \mathrm{~m} * 3.8 \mathrm{~m}$ and 2 antenna clusters are deployed in it; each cluster has 4 antennas. The experiment system is composed as we describe in Section 2.

LMUs are used to process the data received by antenna clusters, and the composition and features of LMU are presented in detail as Figure 9. Each unit has 4 receiving channels, and the signal in each channel is expected to be sampled approximately at the same time. Due to the fact that a small timing error may cause a large mistake during estimation for TDOA localization, it is imperative to precisely synchronize each channel. Through location measurement module, location data can be collected and sent to the location sever for analyzing, and the estimated position is finally calculated by the proposed algorithms. In a word, this design can release the demand of synchronization between base stations and save the cost of network side.

Firstly, we try to locate fixed targets in the conference room by using the deployed system. Figure 10 shows the positioning results of 6 fixed targets. Each picture presents the estimated position distribution of 50 test results, where the blue star is the target (as the center of a blue circle with radius 


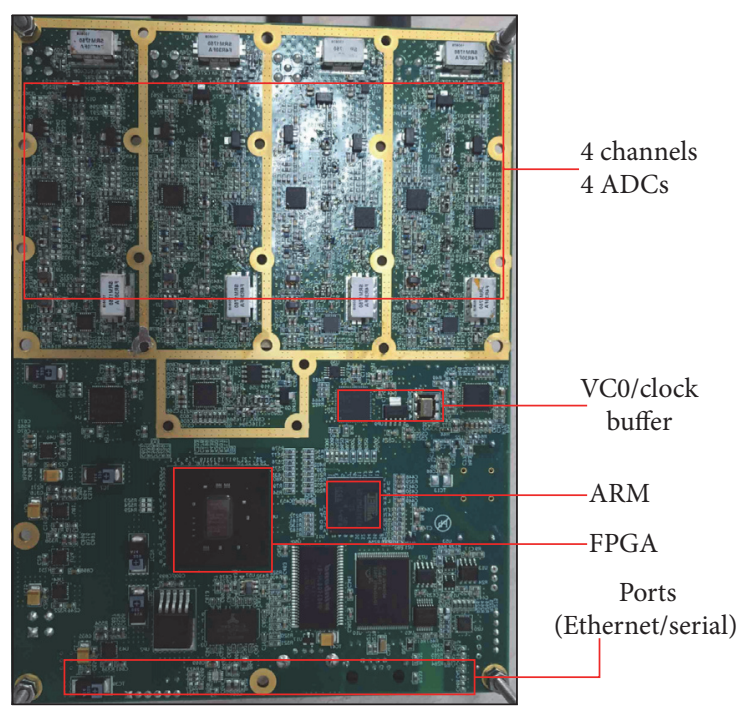

FIGURE 9: The composition of location measurement unit.
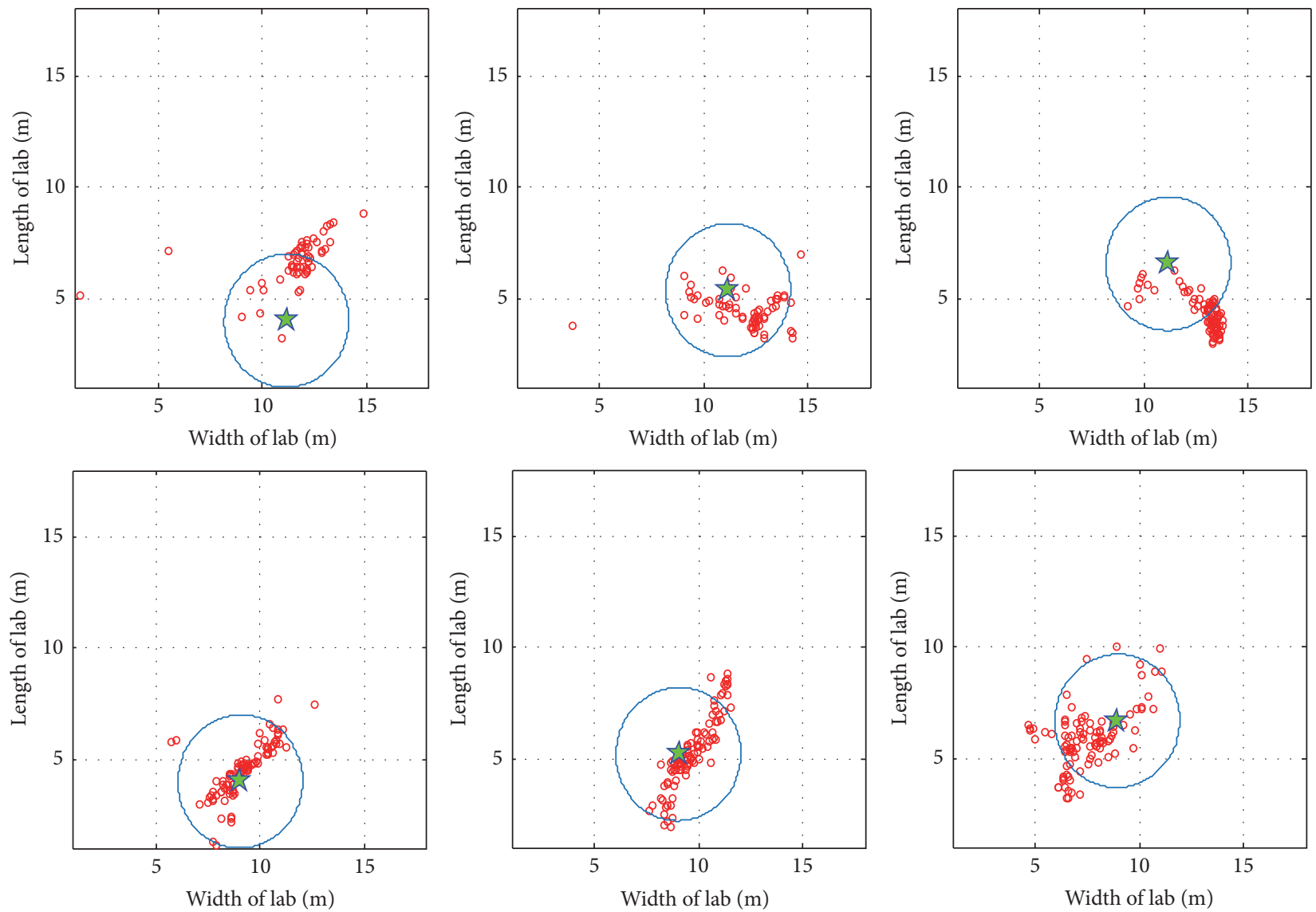

FIGURE 10: Multiple measurement results.

of 3 meters) and the red points are the 50 estimations. The final position results are shown in Figure 11 by aggregating the multiple measurement results.

From the statistic analysis about the positioning results in Table 1, as we can see, the possibility of measurement error less than $3 \mathrm{~m}$ is about $80 \%$. And the maximum estimation error is $3 \mathrm{~m}$, the minimum is $0.47 \mathrm{~m}$, and the average is $1.3 \mathrm{~m}$. Due to the complexity of real environment, the measurement error is larger than the simulation results but still can satisfy the indoor positioning precision. 

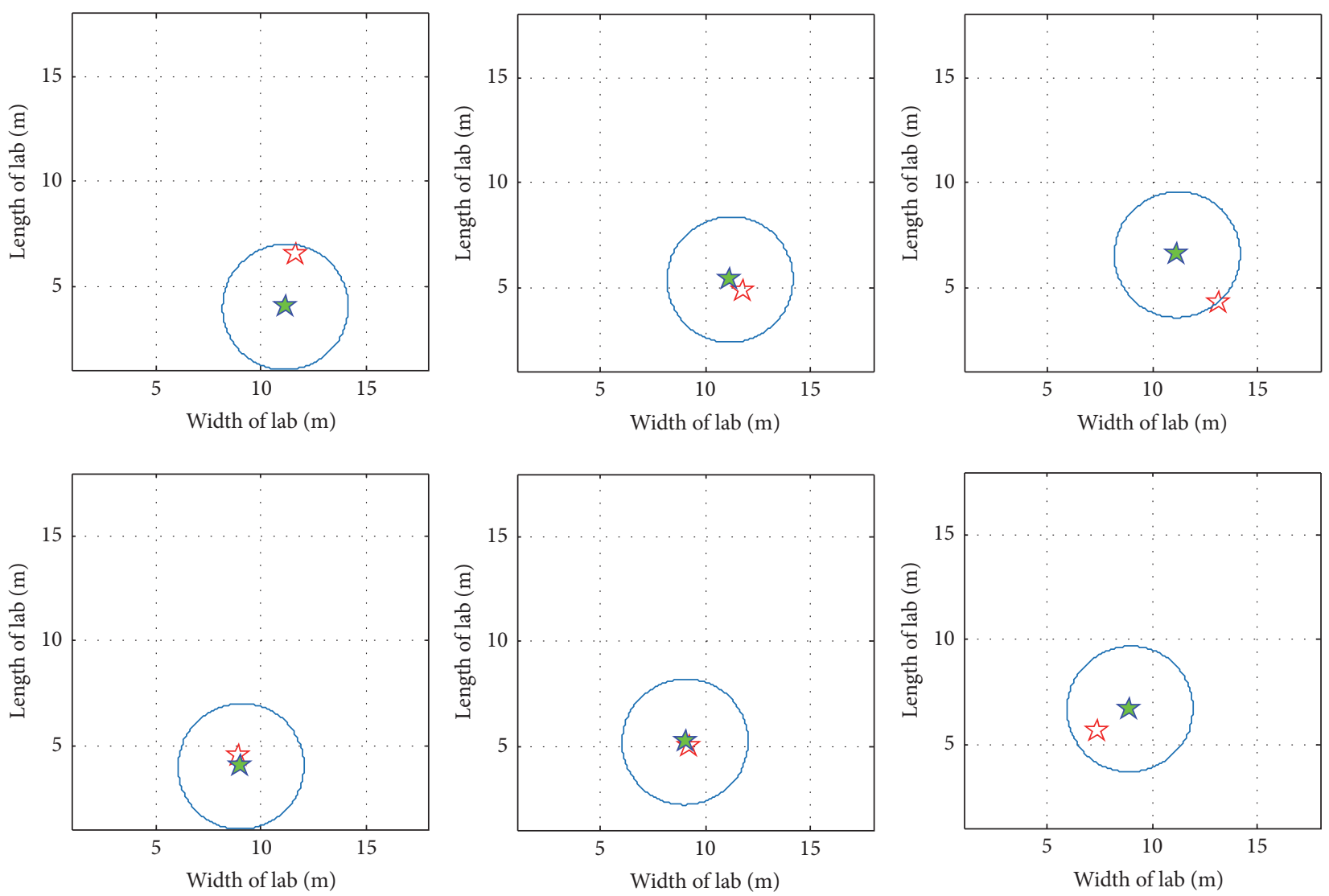

FIGURE 11: Final estimation of position.

TABLE 1: Statistic analysis of measurement results.

\begin{tabular}{lcccc}
\hline Number & $<2 \mathrm{~m}(\%)$ & $<3 \mathrm{~m}(\%)$ & $<4 \mathrm{~m}(\%)$ & $\begin{array}{c}\text { Measurement } \\
\text { error }(\mathrm{m})\end{array}$ \\
\hline 1 & 14.8 & 58.6 & 87.9 & 2.50 \\
2 & 69.1 & 85.3 & 92.7 & 0.86 \\
3 & 26.4 & 77.0 & 100 & 3.00 \\
4 & 85.4 & 95.8 & 100 & 0.47 \\
5 & 72.0 & 88.0 & 100 & 0.53 \\
6 & 49.5 & 83.8 & 95.0 & 1.90 \\
Average & 53.5 & 79.3 & 92.0 & 1.30 \\
\hline
\end{tabular}

Then we testify the UE's path tracing performance; Figure 12 presents the path tracking result of the UE moving at walking speed. The red line denotes the true path, and the blue line is the estimated trajectory. It can be seen that the estimated trajectory is very close to the true path and the measurement error is below $1 \mathrm{~m}$. Comparing with the fixed measurement, the system of dynamic positioning is more stable and more precise. The possible explanation of the result is that UE's movement makes the error furtherly comply with Gaussian distribution, which can increase the precision by averaging multiple measurements.
In addition, both the simulation and test results show that the LTE location system and algorithm have good performance. In order to deal with the complexity of real scene, we improve our algorithm by selecting TDOA and get the final position result by aggregating multiple position points. This method can reduce the influence of multipath.

But our test environment is still relatively simple and hollowness. In a more complex environment, especially with serious multipath like indoor case, our algorithms may not work well. Other improved algorithms or multipath mitigation technologies should be taken into consideration. For example, in cellular communication systems, the hybrid TDOA and the Angle Of Arrival (AOA) location algorithm can reach higher accuracy than traditional TDOA method does.

And terminal-side hybrid locations with WiFi, Bluetooth, or other RF location systems are not precluded to improve the poor precision of wireless location in cellular network [19, 20]. Besides, the assistant positioning technologies of terminal-side equipment like inertial navigation and better filtering algorithms of tracking will be good for location estimation and prediction [21].

\section{Conclusions}

This paper introduces a new user location system in LTE networks with C-RAN architecture. In this system, uplink SRS 


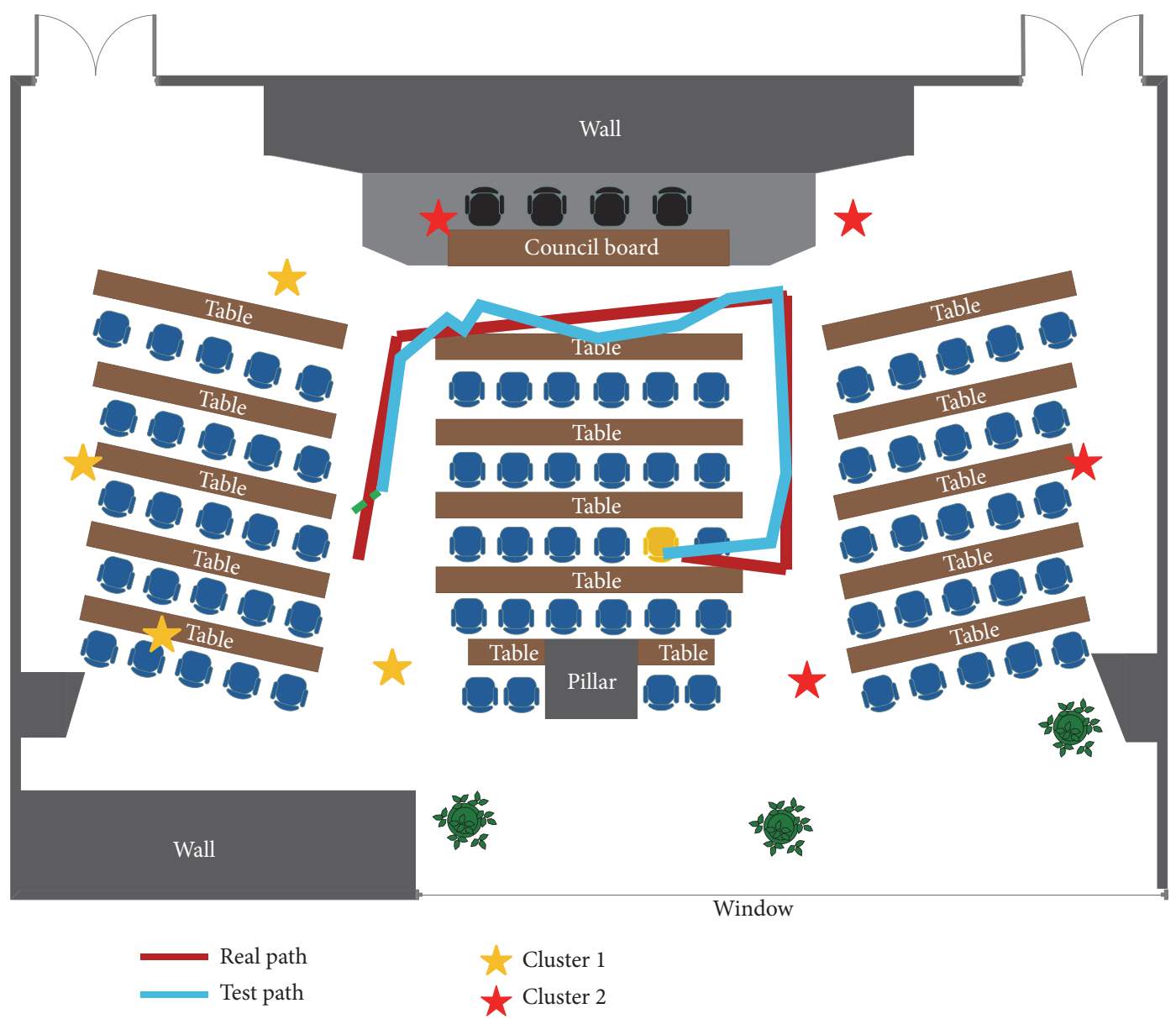

FIGURE 12: The test result of path tracking.

is used as monitoring signals. LMUs are responsible for signal detection and TDOA estimation. The location information server calculates the user's position through the proposed algorithms. Furthermore, based on the basic TDOA algorithm in most location systems, a new location algorithm is raised. It reduces synchronization demands among distributed antennas, which is verified to have a good performance on position estimation. In addition, to avoid the effects of NLOS and multipath overlap, we also propose an improved optimization algorithm by selecting the best TDOAs. Simulation results show that the proposed algorithm has performance gain and improves the efficiency and accuracy of the location system in C-RAN architecture.

\section{Competing Interests}

The authors declare that they have no competing interests.

\section{Acknowledgments}

This work is supported by National Key Research and Development Plan Grants no. 2016YFB0502000. The authors would like to acknowledge the helpful pieces of advice from Project Partner Professor Deng Zhongliang in Beijing University of
Posts and Telecommunications and Professor Liu Huaping, Wang Youxiang, Ph.D., Qiu Jiahui, Ph.D., Ma Yue, Zhang Wenhao, and Chen Yi.

\section{References}

[1] P. J. Voltz and D. Hernandez, "Maximum likelihood time of arrival estimation for real-time physical location tracking of 802.11a/g mobile stations in indoor environments," in Proceedings of the Position Location and Navigation Symposium (PLANS '04), pp. 585-591, April 2004.

[2] C.-P. Yen and P. J. Voltz, "Indoor positioning based on statistical multipath channel modeling," EURASIP Journal on Wireless Communications and Networking, vol. 2011, article no. 189, 2011.

[3] Q. Liu and Y. Ma, "A wireless location system in LTE networks," in Proceedings of the 2nd IEEE International Conference on Consumer Electronics-Taiwan (ICCE-TW '15), pp. 160-161, June 2015.

[4] S. Wei, L. Qi, L. Yiqun, B. Meng, C. Guoli, and Z. Hongke, "Small cell deployment and smart cooperation scheme in dual-layer wireless networks," International Journal of Distributed Sensor Networks, vol. 2014, Article ID 929805, 7 pages, 2014.

[5] S. Sesia, I. Toufik, and M. Baker, LTE-The UMTS Long Term Evolution, John Wiley \& Sons, 2011. 
[6] H. Wu, Y. Chen, N. Zhang et al., "The NLOS error mitigation joint algorithm in hybrid positioning system combining DTMB and GPS," in Proceedings of the China Satellite Navigation Conference (CSNC '12), pp. 287-296, Guangzhou, China, May 2012.

[7] W. Kim, J. G. Lee, and G.-I. Jee, "The interior-point method for an optimal treatment of bias in trilateration location," IEEE Transactions on Vehicular Technology, vol. 55, no. 4, pp. 12911301, 2006.

[8] R. Ye and H. Liu, "UWB TDOA localization system: receiver configuration analysis," in Proceedings of the International Symposium on Signals, Systems and Electronics (ISSSE '10), pp. 205208, September 2010.

[9] Y. Zhou, L. Jin, C. Jin, and A. Zhou, "FIMO: a novel WiFi localization method," in Web Technologies and Applications, vol. 7808 of Lecture Notes in Computer Science, pp. 437-448, Springer, Berlin, Germany, 2013.

[10] A. Catovic and Z. Sahinoglu, "The Cramer-Rao bounds of hybrid TOA/RSS and TDOA/RSS location estimation schemes," IEEE Communications Letters, vol. 8, no. 10, pp. 626-628, 2004.

[11] M. R. Gholami, S. Gezici, and E. G. Strom, "A concave-convex procedure for TDOA based positioning," IEEE Communications Letters, vol. 17, no. 4, pp. 765-768, 2013.

[12] R. Ye, S. Redfield, and H. Liu, "High-precision indoor UWB localization: technical challenges and method," in Proceedings of the IEEE International Conference on Ultra-Wideband (ICUWB '10), pp. 1-4, IEEE, Nanjing, China, September 2010.

[13] B. Kempke, P. Pannuto, and P. Dutta, "Harmonia: wideband spreading for accurate indoor RF localization," in Proceedings of the 1st ACM MobiCom Workshop on Hot Topics in Wireless (HotWireless '14), pp. 19-23, New York, NY, USA, September 2014.

[14] O. Simeone, A. Maeder, M. Peng, O. Sahin, and W. Yu, "Cloud radio access network: virtualizing wireless access for dense heterogeneous systems," Journal of Communications and Networks, vol. 18, no. 2, Article ID 7487951, pp. 135-149, 2016.

[15] J. Wu, Z. Zhang, Y. Hong, and Y. Wen, "Cloud radio access network (C-RAN): a primer," IEEE Network, vol. 29, no. 1, pp. 35-41, 2015.

[16] R. Wang, H. Hu, and X. Yang, "Potentials and challenges of C-RAN supporting multi-RATs toward 5G mobile networks," IEEE Access, vol. 2, pp. 1200-1208, 2014.

[17] C. H. Knapp and G. C. Carter, "The generalized correlation method for estimation of time delay," IEEE Transactions on Acoustics, Speech, and Signal Processing, vol. 24, no. 4, pp. 320327, 1976.

[18] H. Liu, H. Darabi, P. Banerjee, and J. Liu, "Survey of wireless indoor positioning techniques and systems," IEEE Transactions on Systems, Man \& Cybernetics Part C: Applications and Reviews, vol. 37, no. 6, pp. 1067-1080, 2007.

[19] S. He and S.-H. G. Chan, "Wi-Fi fingerprint-based indoor positioning: recent advances and comparisons," IEEE Communications Surveys and Tutorials, vol. 18, no. 1, pp. 466-490, 2016.

[20] S. Bozkurt, S. Günal, U. Yayan, and V. Bayar, "Classifier selection for RF based indoor positioning," in Proceedings of the 23rd Signal Processing and Communications Applications Conference (SIU '15), pp. 791-794, IEEE, Malatya, Turkey, May 2015.

[21] K. He, Y. Zhang, Y. Zhu, W. Xia, Z. Jia, and L. Shen, "A hybrid indoor positioning system based on UWB and inertial navigation," in Proceedings of the International Conference on Wireless Communications and Signal Processing (WCSP '15), pp. 1-5, Nanjing, China, October 2015. 

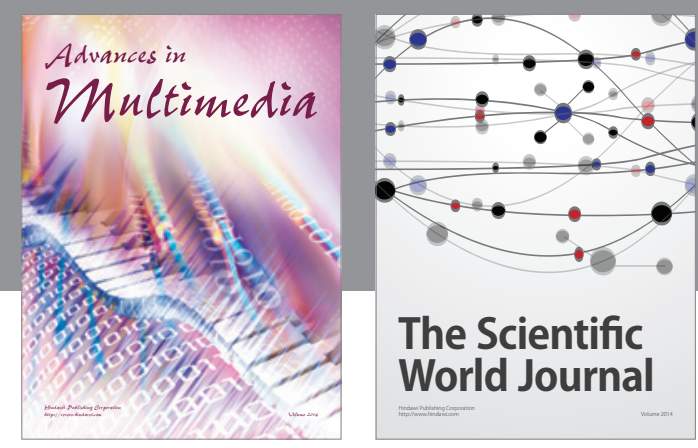

The Scientific World Journal
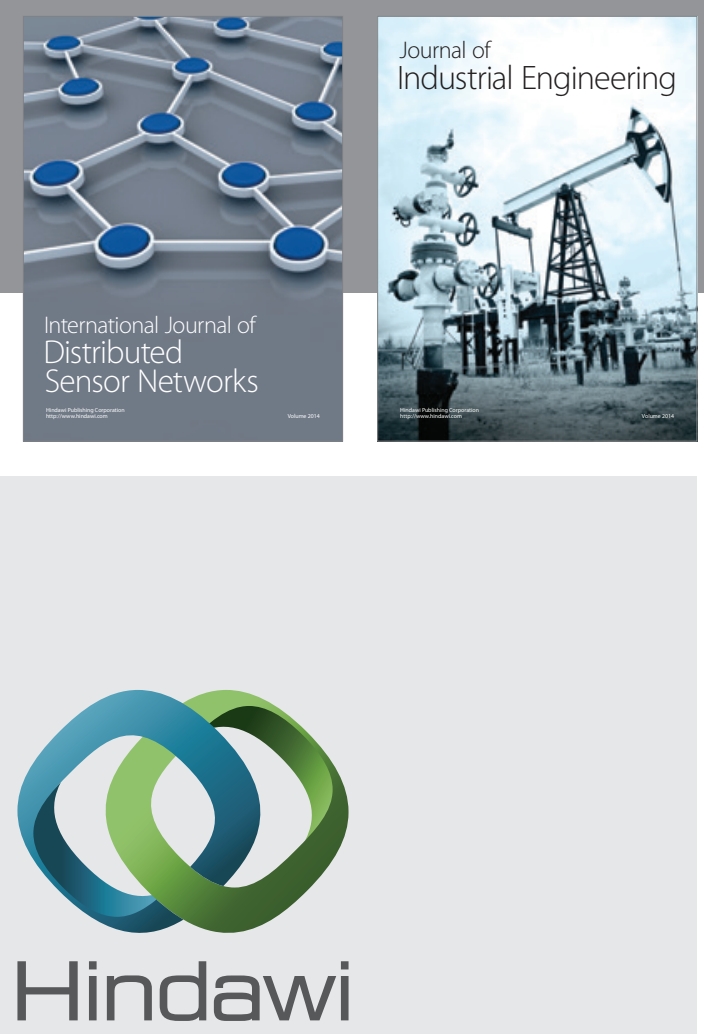

Submit your manuscripts at

https://www.hindawi.com

\section{Computer Networks} and Communications
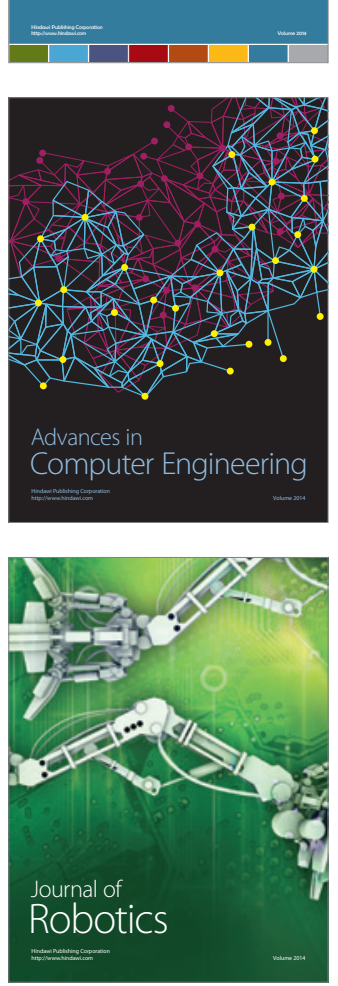
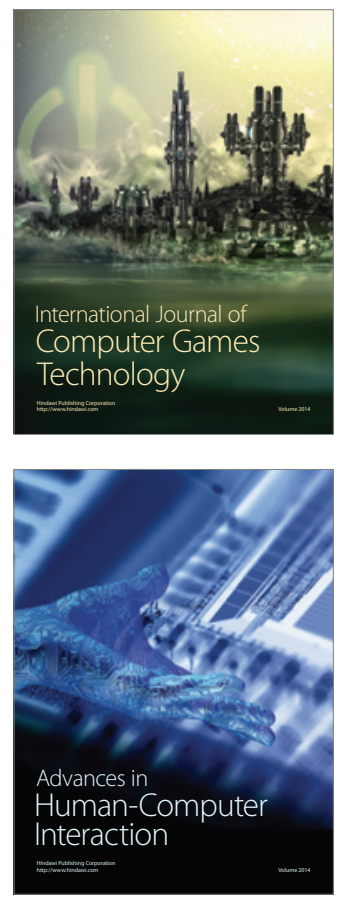
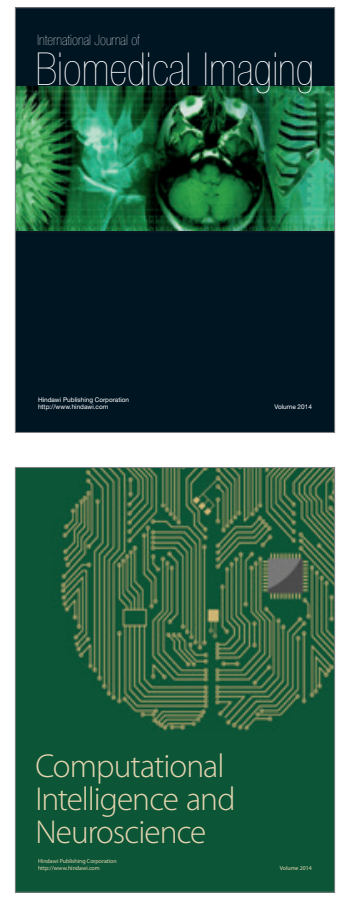
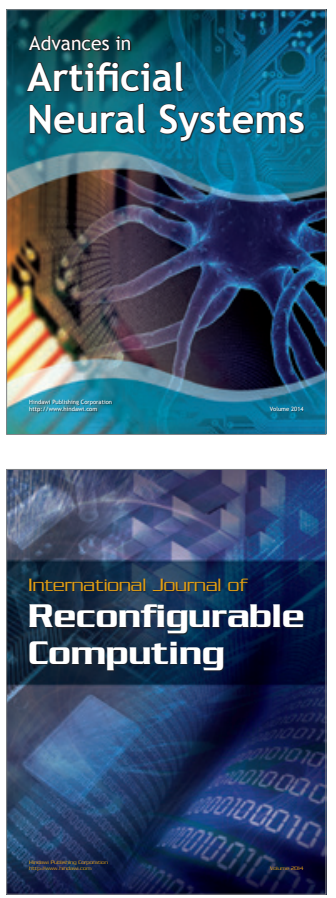
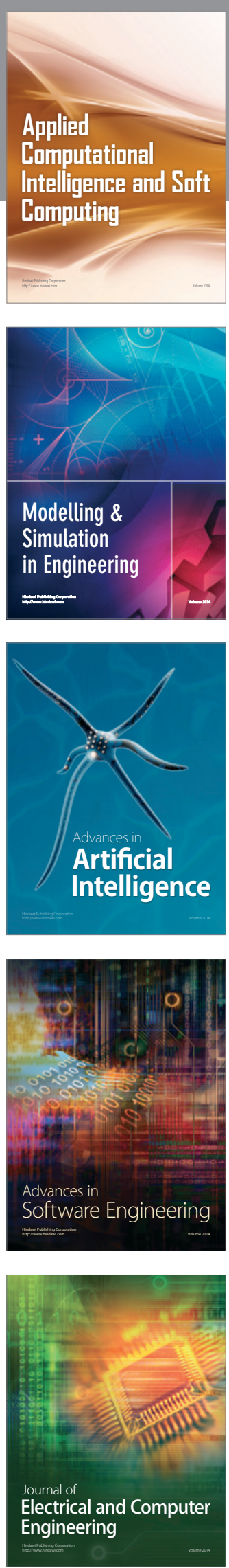